\title{
Optimizing the Passenger Throughput at an Airport Security Checkpoint Ying $\mathrm{Xu}$ and Jingwen Hua \\ North China Electric Power University, Beijing, 102206 \\ alanncepu@foxmail.com
}

\begin{abstract}
Keywords: Passenger throughput; Queuing theory; Waiting time; A win-win goal for cost and efficiency
\end{abstract}

\begin{abstract}
At present, there exists a common phenomenon that the waiting time for passengers at the security checkpoint is too long and the cost is too high in solving the sudden peak flow of people in the majority of airports in the world. To optimize the passenger throughput, we have set up a model which considered the cost of official staff and customer satisfaction as overall objectives. The model based on queuing theory shows that how many check-in counters should be opened with the different flow of passengers. It has effectively achieved a win-win goal for cost and efficiency and has reduced variance in wait time with opening available counters when a sudden peak flow hit.
\end{abstract}

\section{Queuing Analysis of Steady State Serial Queuing Networks}

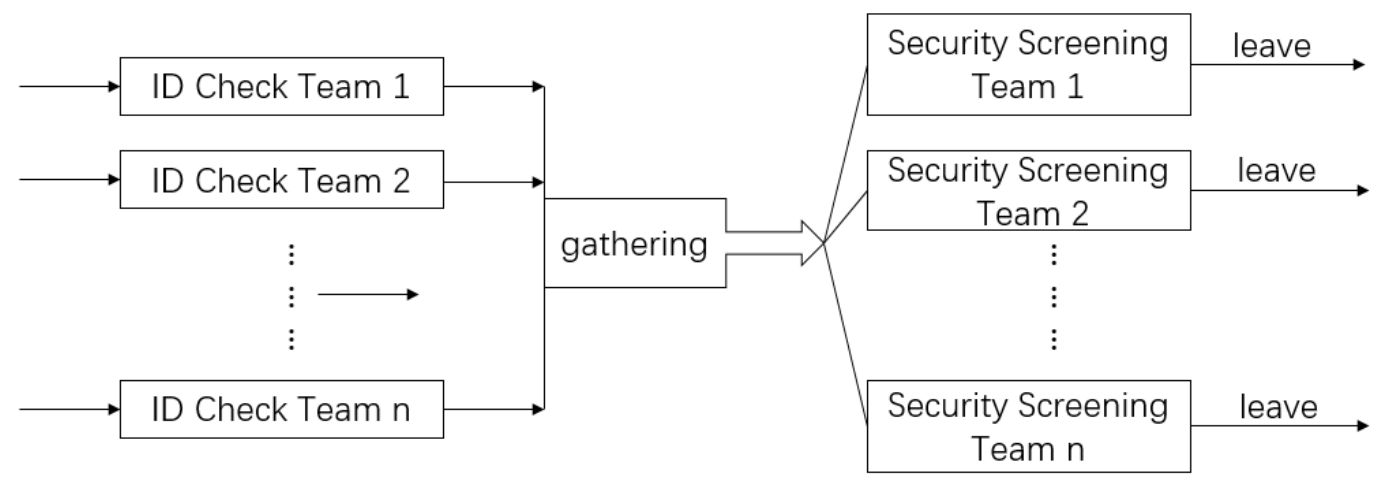

Figure1.1 M/ M/k system

If the queuing system is an $\mathrm{M} / \mathrm{M} / \mathrm{k}$ system and the utilization rate is less than 1 , its output rate is equal to the input rate. In this case, we call it the steady-state queuing system, and we call the network composed of the steady queuing system a steady-state queuing network. The passenger flow, as a queuing network, is an open-loop network called the Jackson network. If the arrival time of the first queuing system obeys exponential distribution with parameter $\lambda$, the service time of each stage is exponentially distributed, and each phase has unlimited waiting space, then the queuing system arrival time interval is subject to exponential distribution with parameter $\lambda$ in various stages.

\section{Analysis of Check in}

First of all, we analyze the queuing system of the check-in system. The check-in system is the first queuing system in the whole queuing network. It is also the most complicated link and the analysis method is representative. The passenger input of the check-in system is related to the flight schedules, the number of passengers arriving in the flight and the cumulative distribution curve of the passengers. The number of passengers arriving at any time is the sum of arriving times of each flight. The arrivals of passengers on each flight are independent of each other. At some point, the passenger arrival rate of a check-in system is the sum of the passenger arrival rates on $\mathrm{L}$ flights at the same time.

$$
\lambda(t)=\lambda 1(t)+\lambda 2(t)+\cdots+\lambda l(t)
$$


Suppose the service system has k service desk. The service desk rates are equal to $\mu$. The total service rate is equal to $\mathrm{k} \mu$. We define the utilization (service intensity) $\rho$ as the ratio of the total arrival rate of the queuing system to the total service rate, and we require that the average utilization $\rho$ is less than 1. The flight arrival time is divided into several periods, and the passenger arrival rate in each time period is regarded as constant, then the total arrival rate of passengers of each flight time is also regarded as constant. According to the $M$ / $M$ / k queuing system equation, if the passenger arriving rate is $\lambda$ person / $\mathrm{min}$, the service rate of each counter check-in is $\mu$ person $/ \mathrm{min}$, the number of open counters is $\mathrm{K}$, the passenger check-in queue average length is equal to

$$
L_{k}=\frac{\rho^{k+1}}{k k !(1-\rho / k)^{2}}\left[\sum_{n=0}^{k-1} \frac{\rho^{n}}{n !}+\frac{\rho^{k}}{k !(1-\rho / k)}\right]^{-1}
$$

Among them, $\rho=\lambda / \mu, \quad \mathrm{k}>1, \quad \mathrm{k}>\rho$ 。

Simplified equation is:

$$
\mathrm{L}_{\mathrm{q}}(\mathrm{k})=\frac{\rho^{\mathrm{k}+1}}{(\mathrm{k}-\rho)\left(\sum_{\mathrm{n}=0}^{\mathrm{k}-1} \mathrm{C}_{\mathrm{n}} \rho^{\mathrm{n}}\right)}
$$

For the common case, $\mathrm{k}=2$ :

$$
\mathrm{L}_{\mathrm{q}}(\mathrm{k}=2)=\frac{\rho^{3}}{(2-\rho)(2+\rho)}
$$

The general expression of the coefficient $C_{n}(n<k-1)$ for any term in the denominator is:

$$
\mathrm{C}_{\mathrm{n}=}(\mathrm{k}-1) ! \frac{(\mathrm{n}-\mathrm{k})}{\mathrm{n} !}
$$

The average queuing time for passengers is:

$$
\mathrm{W}_{\mathrm{q}}=\mathrm{L}_{\mathrm{q}} / \lambda
$$

The probability of a passenger arriving at the queue is equal to the average busy probability of the attendant which is given by Eq.1.

$$
C(\rho, k)=\frac{\rho^{k}}{k !\left(1-\frac{\rho}{k}\right)}\left[\sum_{n=0}^{k-1} \frac{\rho^{n}}{n !}+\frac{\rho^{k}}{k !\left(1-\frac{\rho}{k}\right)}\right]^{-1}=\frac{k-\rho}{\rho} L_{q}
$$

The average queue length, the average queue time and the probability of arriving passengers need to queue are three important indexes which affect the passenger satisfaction. The following curve shows that, when $\boldsymbol{\rho}$ is near the number $\mathrm{k}$ of the check-in counter, the probability of the crew and the average length of the passenger queue are jumped.

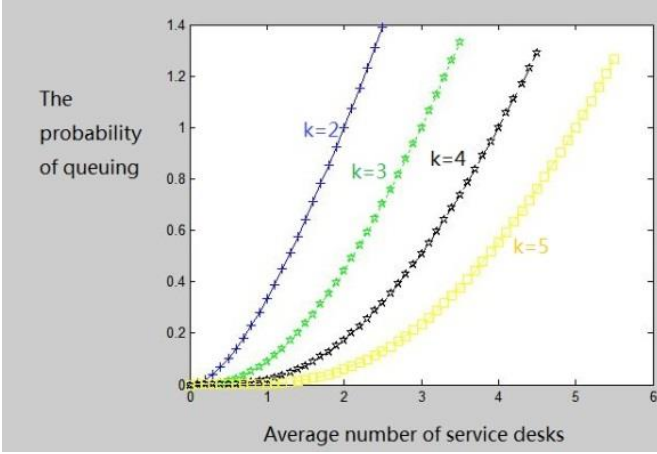

Figure 1.2.

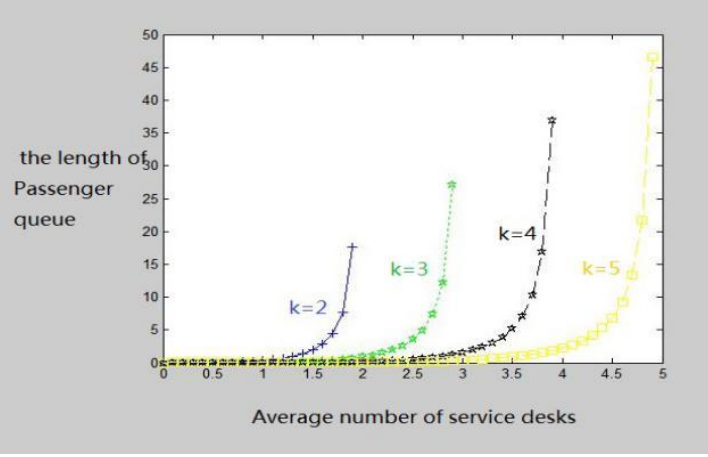

Figure 1.3.

\section{Check - in Counter Distribution Model and Algorithm}

First, the objective function is discussed. For public counters, the check-in will not distinguish 
between airlines and flights. Passengers can queue up at any time, waiting for check-in. In the case of knowing arrival rates and per-desk service rates, the metrics for queuing systems can be calculated as

$$
\operatorname{maxz}=p_{s} C(\rho, k) k \mu+q_{w}\left(l_{c}-L_{q}\right)-c_{s} k
$$

In the equation, $P_{s}$ is a system of services generated by the benefits of passengers, $q_{w}$ is the value of passenger satisfaction coefficient of value-added efficiency, $c_{s}$ is the cost per unit time to open a service desk.C $(\rho, \mathrm{k})$ is the busy probability of the crew. Its size reflects the labor intensity of the crew, and is calculated by Eq.1. The meaning of other parameters is given by Eq.4. Constraints include the requirement that the passenger queue length should be no more than $l_{c}$.

$$
L_{q} \leq l_{c}
$$

$\mathrm{l}_{\mathrm{c}}$ is the passenger queue length standard among them. As discussed above, $\mathrm{L}_{\mathrm{q}}$ is the average queue length, calculated by the Eq.4. The significance of this constraint is that if the average passenger length is less than the length of the standard, the airport will receive passenger value-added benefits; if the length is more than the length of this standard, the airport reputation will damage because of reducing passenger satisfaction.

Other constraints are as follows:

$$
\left\{\begin{array}{l}
\mathrm{k} \leq \mathrm{C} \\
\mathrm{k}>\rho \\
P_{\text {min }} \leq C(\rho, k) \leq P_{\text {max }}
\end{array}\right.
$$

$\mathrm{C}$ is the number of open check-in counters among them. The first constraint is the resource limit, which means that the number of counters allocated cannot exceed the number of open counters. The second constraint indicates that the number of counters allocated cannot be less than the average number of counters used. Since the queuing theory shows that the average number of service stations $\rho$ is close to $\mathrm{k}$, the length of the passenger queue will approach infinity, which means that the probability of the average busy is closer to unity. The third constraint indicates that the busy probability of the crew should be within a reasonable range. Too much busy probability may cause work errors and low efficiency. Too small busy probability may decline the utilization of resources. Computational practice suggests that this range of the third constraint should be larger, such as $[0.45,0.75]$, otherwise it may cause the problem to be infeasible. In the objective function, since $q_{w} 、 l_{c}$ are constants, they can be eliminated.At this time, $\mathrm{q}_{\mathrm{w}} 、 \mathrm{l}_{\mathrm{c}}$ are equivalent to the cost of passenger queuing, so we can get the check-in queuing system service station optimization model as shown

$$
\begin{cases}\operatorname{maxz} & =p_{s} C(\rho, k) k \mu-q_{w} L_{q}-c_{s} k \\ \text { s.t. } & \mathrm{k} \leq \mathrm{C} \\ & \mathrm{k} \geq[\rho]+1 \\ & L_{q} \leq l_{c} \\ & P_{\min } \leq C(\rho, k) \leq P_{\max }\end{cases}
$$

\section{Solving the Model}

Eq.11 is a nonlinear integer programming problem, but it is difficult to solve it. However, because the variable range of decision variable $\mathrm{k}$ is not big, it can be solved by heuristic algorithm and enumeration method. First, only the objective function is considered, and the marginal analysis method is used to find that the number of check-in counters $\mathrm{k}^{*}$ of the objective function maximum should satisfy the inequality as shown in Eq.12.

$$
\frac{c_{s}}{\mu p_{s}} \leq \frac{q_{w}}{p_{s}} \rho\left(W_{q}\left(\mathrm{k}^{*}-1\right)-W_{q}\left(\mathrm{k}^{*}\right)\right)-k^{*} C\left(\rho, \mathrm{k}^{*}-1\right)
$$




$$
\frac{c_{s}}{\mu p_{s}} \geq \frac{q_{w}}{p_{s}} \rho\left(W_{q}\left(\mathrm{k}^{*}\right)-W_{q}\left(\mathrm{k}^{*}+1\right)\right)-k^{*}\left[C\left(\rho, \mathrm{k}^{*}\right)-C\left(\rho, \mathrm{k}^{*}+1\right)\right]+C\left(\rho, \mathrm{k}^{*}+1\right)
$$

Then we test whether $\mathrm{k}^{*}$ satisfies the constraints of the optimization model. If it is satisfied, it is the optimal solution of the problem; if not satisfied, then we see that it violates which constraints, and then under $\mathrm{L}_{\mathrm{q}}$ and $\mathrm{C}(\rho, \mathrm{k})$ with $\mathrm{k}$ increases and decreases the law for $\mathrm{k} *$ plus or minus 1 adjustment And get the optimal solution of the problem .To compute the solution satisfying the inequality, Figure 1.3 can be applied. First, $\mathrm{k}^{*}$ should be in the vicinity of $\rho$, and then refer to Figure 1.2, respectively, we can get the crew's busy probability of the queuing system $C(\rho, k)$ and passenger queue length $L_{q}$, and Substitute them to the right of the equation, determine what size of $\mathrm{k}$ could meet equation, so that the number of check-in counters with the optimal objective function can be obtained.

\section{References}

[1]Yun Yang, Establishment and optimization of security facilities in small and medium sized Airports [J].Electronic world, 2015(15):195-196.

[2] Zhenwu Zhao, Jianjun Ma, Development trend and research of civil aviation airport security queuing system [J]. Integrated Transport, 2016 (10): 64-69.

[3] Gu Yang, Zheng Min, Zhou hang, et al, Study on dynamic allocation method of airport security resources [J]. Aviation Computing Technology, 2016, 46 (5): 67-72.

[4] Jiang Xinxin, Zhou hang, Cai Bingqing, Research on the layout and process optimization of terminal security. [J]. Aeronautical Computing Technology, 2015 (3): 25-29.

[5] Jia Guoyang, Research on Optimization of passenger security service process based on LCIOWF. [D]. Harbin Institute of Technology, 2015.

[6] Zhang Yong, Wang Xiaobin. Analysis and solution of the problem of centralized safety inspection system [J]. Mechanical and Electrical Product Development and Innovation, 2015, 28 (1): 105-107.

[7] Ma Jun. Application of virtual queuing in civil aviation security inspection system [J]. Science and Technology, 2016 (19): 34-35.

[8] Li Yu, Application of virtual queuing in civil aviation security inspection system, [J]. Science and Technology, 2017, 27 (8)

[9] Meng Rui. Study on Optimization Design of security system in capital airport [J]. China Security, 2014 (20): 90-93.

[10] Zhao Y, Jiang H. Design and implementation of Railway Station Freight Transportation Inspection Centralized Safety Monitoring System [J]. Railway Computer Application, 2009. 